\title{
Using Face Quality Ratings to Improve Real-Time Face Recognition
}

\author{
Karl Axnick, Ray Jarvis, and Kim C. Ng \\ Monash University, Wellington Rd, Clayton, VIC, 3800, Australia \\ \{karl.axnick, ray.jarvis,kim.c.ng\}@eng.monash.edu.au
}

\begin{abstract}
A Face Quality Rating (FQR) is a value derived from a face image that indicates the probability that the face image will be successfully recognized by a specific face recognition method. The FQR can be used as a pre-filter in real-time environments where thousands of face images can be captured every second by multiple surveillance cameras. With so many captured face images, face recognition methods need to strategically decide which face images to attempt recognition on, as it is prohibitively difficult to attempt recognition on all of the images. The FQR pre-filter optimizes processor time utilization resulting in more people being recognized (faster and more accurately) before they leave the surveillance cameras' views. We generate FQR values using Multiple Layered Perceptron (MLP) neural networks. We then use these MLPs in a real-time environment to experimentally prove that FQR pre-filtering improves the speed and accuracy of any real-time face recognition method...
\end{abstract}

\section{Introduction}

FQR pre-filtering is ideal for real-time face recognition applications [1]. These applications include public environments such as airports, train stations and streets under CCTV surveillance. These are very difficult face recognition environments because there are multiple cameras, multiple entry and exits points, multitudes of targets and vastly varying poses, expressions, occlusions, scales and illumination levels. This is arguably the most difficult environment for face recognition 2. Systems need to quickly decide which face image to recognize out of thousands, before any number of people leave the scene or enter sensitive areas. The time critical elements, the multitudes of redundant data and the sequence dependent outcomes mean that classical recognition methods [3] are ineffective and readily overwhelmed (refer to Fig. 5).

Face recognition has been an intense and extensive area of research over the past 20 years [4. Many unique approaches have been adopted outside of the standard "detect a face and recognize it" systems, such as "divide and conquer" strategies [5], cascading filters [6] and pre-recognition normalization [7. These approaches have enjoyed considerable success in conventional face recognition settings, but they all rapidly fail in difficult real-time environments as the number of people in the scene increases (refer to Fig. 5 ). This failure can be attributed to

T. Wada, F. Huang, and S. Lin (Eds.): PSIVT 2009, LNCS 5414, pp. 13-24, 2009.

(C) Springer-Verlag Berlin Heidelberg 2009 
the decision in the approaches that if a face is detected, it must have recognition attempted. Although this decision seems logical given the field, unless the face recognition approach is $100 \%$ accurate the decision is going to waste valuable processor time trying to recognize every single face the system detects. FQR pre-filtering enables a system to intelligently allocate recognition cycle times for only those face images that offer the best chance of recognition success. It is important to note that recognition success includes correctly classifying a face as not being present in the database. Without FQR pre-filtering, face recognition methods can repeatedly waste recognition cycles trying to recognize people who simply cannot be recognized because they are not in the database.

FQRs are a probability measure only and other factors besides face quality can affect recognition success. For example, the size, composition, and inter-class variations of the databases play a role in the success of recognition methods that use them. FQR pre-filtering is a powerful tool that is best used in conjunction with other tools that also help increase recognition speed and accuracy such as "divide and conquer" strategies [5].

Xiong [8] improves automatic database image acquisition in unconstrained environments by using Fisher's Linear Discriminant to measure the separability of classes in the database. Whenever the system detects a face, it tries to automatically add this to the database to increase system robustness. If the addition would not increase class seperability or is redundant, then the image is ignored. Subasic [9] helps improve database image acquisition in constrained environments by observing International Civil Aviation Organization (ICAO) rules. ICAO rules are a collection of simple tests such as requiring normalized eye widths from acquired images. These papers are examples of how face/image quality metrics can be used to improve face recognition results by improving database creation. Our FQR method can be used in a similar manner.

It is important to note that during FQR assisted video surveillance all of the people who are detected in the scene have their best images to date stored, and all of the people will eventually be subjected to recognition using their best image, whether or not their best image has a high FQR. We delay using valuable processing time on low FQR images, because on average a better FQR image will eventuate if we wait. In the meantime, that processing time can be more efficiently used on other peoples' more highly ranked FQR images. If a person's best image has a low FQR but must undergo recognition, we increase the required recognition threshold for a match to ensure accurate results from that difficult low FQR image. It should be noted that the detection of a low FQR value for an input face can in effect lead to a decision to classify the input as a possible "reject class". Recognition of reject classes has a long history in pattern recognition [10] and this further validates the FQR pre-filtering approach.

The format of this paper is as follows; Section 2) illustrates our methodology and its advantages; Section 3) explains how an FQR can be learned using an MLP neural network $(\mathrm{NN})$, and proves that the rating can predict the probability of recognition success; Section 4) explains and reports on the experiments that use the MLP NN described in section 3; and finally Section 5) concludes our findings. 


\section{Methodology}

A FQR is obtained by using an MLP NN that has been trained with a specific face recognition method and a training database. Training involves exposing a face image to the MLP and using back propagation to reinforce correct predictions of recognition success at the output depending on whether the recognition method correctly classified the face image. This training is time consuming, but it is done off-line and only once for each face recognition method that requires a FQR. The MLP learns how to detect many sub features in the images and uses their presence or absence as recognition predictors. We currently use the MLP FQR pre-filter after the normalization stage and before the face recognition stage in the recognition system (refer to Fig. 1).

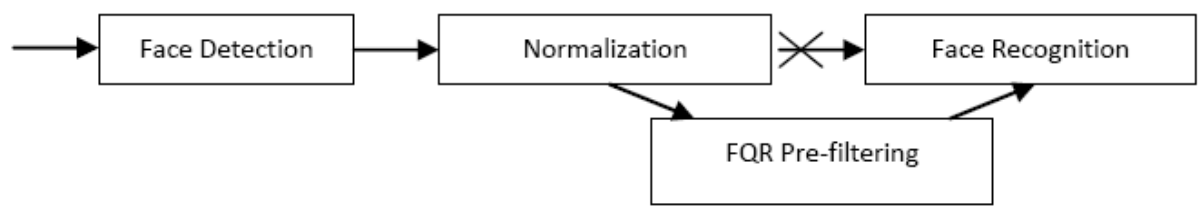

Fig. 1. The new face recognition system tests the face quality before recognition is attempted

FQR pre-filtering at this stage offers many advantages over conventional face recognition systems:

1. The final face recognition stage can be avoided for a face image with a low FQR. This saves on the recognition-time expense for an image that is probably unrecognizable.

2. The final face recognition stage can be delayed until a better quality image of that face is captured, thereby improving the probability of success.

3. FQRs could be used to automatically select face recognition thresholds and/or parameters in response to detected FQRs to improve face recognition confidence.

4. While a detected person is still unrecognized, whether in the scene or not, the face recognition system will only need to store a small set of high FQR images for that person (for recognition at a later time). Without FQRs the system would have to store every single image of the person to optimize the chance of recognizing at least one of the input images (an undesired situation especially with many camera's, thousands of frames, and hundreds of people moving quickly through a scene).

5. By attempting to recognize the highest quality faces in a scene first, we quickly remove unrecognized people from the "to do" list of a crowded scene. This makes it less likely that people can leave the scene or enter a sensitive area before having recognition attempted. A person who is difficult to recognize will not "hog" the processor time while many potentially easily recognized people move unrecognized through the scene. 
6. Even if face recognition is always attempted regardless of a person's FQR value, the FQR value can: a) generate a confidence value in the match score, b) cause the required threshold for successful matches to be scaled up, and c) help explain if a target's input image fails to be recognized because the target is not in the database or because the target's input image is too poor in face quality to match with the target's database image.

Many face recognition systems rely on face detection [1] as a quality check. However, face detection is not designed to rank face images against each other, nor is face detection designed to detect key features in faces that certain face recognition methods rely on. Face detection methods are "plug and play" in operation for most face recognition methods, whereas FQR pre-filtering is specifically trained for use with the face recognition method for which it is paired.

FQR values are in no way directly related to image quality. An image with blurred lines as well as salt and pepper noise would probably be considered a poor quality image, but if the image clearly shows a sub feature that the utilized face recognition method finds strongly salient, then the low quality image would have a high FQR. Conversely, a well focused high-resolution image that would normally be considered high quality may have a low FQR value if the person in the photo is wearing sunglasses and the utilized face recognition method relies heavily on the eyes for recognition success.

\subsection{Learning the FQR Value}

To learn FQR values for any given face recognition method (and its associated database) a MLP is used. The MLP is trained using back propagation and simulated annealing [12. The input layer consists of a $1 \mathrm{D}$ vector of the normalized grayscale values for the current target image. The desired output from training is a single value predicting whether or not the target image will be successfully recognized. The MLP does not try to recognize the face whilst getting the FQR.

During training the MLP will slowly learn certain image sub features. Unfortunately, due to MLP's being black boxes it is difficult to be aware of the specifics of these sub features so human observations cannot evaluate the use/robustness of these sub features independently from the system results. What can be evaluated however is the fact that the MLP can use the presence or absence of learned image sub features to generate a single value output that gives the likelihood of the current target image being correctly recognized. In other words the MLP can generate the image's FQR value.

The parameters for the MLP were 64 x 64 inputs, 64 x $64+64$ hidden nodes and 1 output node. However, any NN type, any training method, and any set of parameters could have been used for our purposes as long as a reasonably accurate FQR value for any target image can be learned.

\subsection{Training Data}

The CMU database [13] was used along with our own 3D database [14] (we used the $3 \mathrm{D}$ face models to generate multiple $2 \mathrm{D}$ face images from many perspectives). 
The databases were combined to ensure no bias was found in the results. The individual databases were very effective when used on their own. As well as using real images from the combined database, 10 virtual images of every real image were generated. The virtual images corrupted the originals with various high probability real world degradations such as white noise, occlusions, scale changes, translation changes and pose changes to name a few. These degradations were randomly present or absent in each virtual image. When the images were degraded this was done to a random degree (e.g. anywhere from $20 \%$ to $60 \%$ of the image pixels could be replaced by salt and pepper noise).

Our training database has 3 images per person: 2 frontal images with different expressions (normal and shocked) and 1 profile image. This gave us a total of 270 real images in the training database. We also used a test set for monitoring the training process. The test set consists of: 1 frontal image (with an angry expression) and 1 profile image (not the same as the one used in the training database), giving us a total of 180 real images in the test set.

Ten virtual images were generated for each of the real images in both sets. This gave us a total of 2970 training images and 1960 test images. We used excessive amounts of virtual images because the random degradations seem to (based on experimentation) more readily expose image sub features to the MLP that are critical for the face recognition being used.

\subsection{The Training Process}

It is necessary to select a face recognition method to train the MLP with. We decided to use a $2 \mathrm{D}$ geometric salient feature point (SFP) method 15 and a modified 2D PCA holistic method [16]. We used both holistic and geometric methodologies to prove that FQR pre-filtering can improve the performance of either methodology for face recognition.

FQR pre-filtering will improve real-time performance rates for a face recognition method, no matter how effective that face recognition method is by itself. Any existing face recognition method can be used and improved by our FQR pre-filtering method in both its speed and accuracy for real-time environments. These claims are based on experiments in the next section.

We trained each MLP in parallel with its partnered face recognition method (i.e. one MLP for the SFP method and one for the PCA method). After each face recognition method finished recognizing an input, we used the success of the result to train the MLP which was attempting to predict that success. If the face recognition method succeeded, and the MLP had an output greater than " 0.5 " then the contributing links would be re-enforced through back propagation. If the MLP instead had a negative prediction (i.e. less than " 0.5 ") then the contributing links would have their weights reduced. This process was re-iterated for all of the training images. After each epoch of training, the error level of the system was evaluated. If the error level was less than $10 \%$ we would then test the MLP on the test set without training. If the MLP achieved a success rate greater than $90 \%$ on the test set, then training was considered complete. Otherwise a fresh set of virtual training images were generated and the supervised training cycle was repeated. 


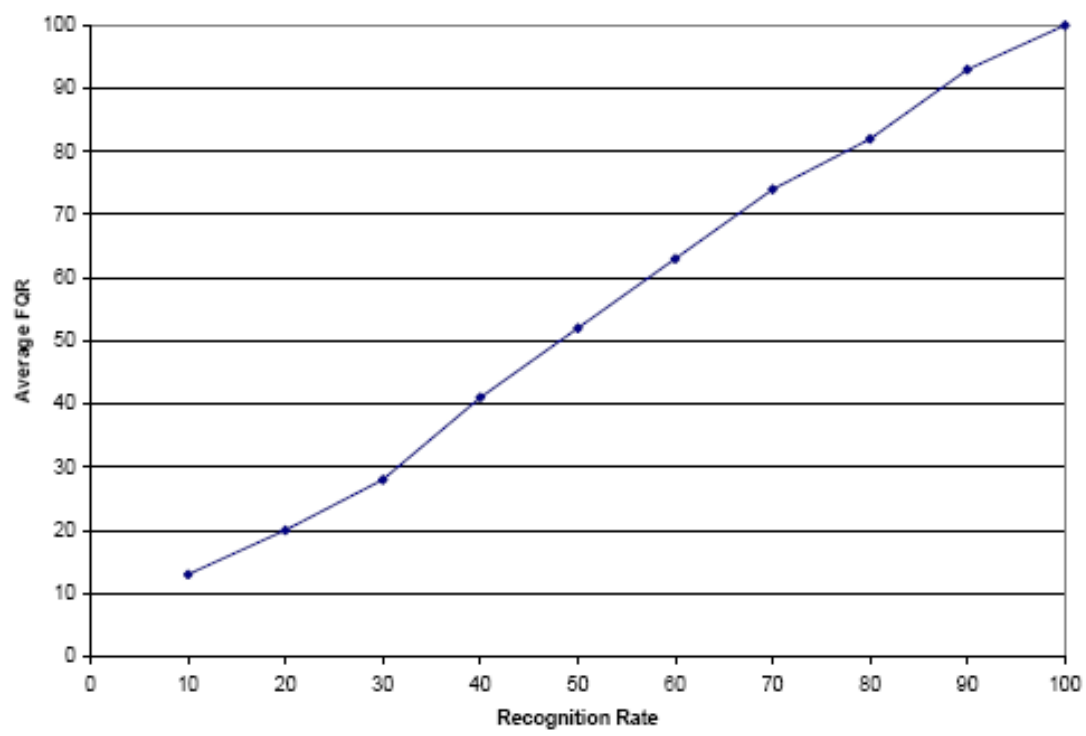

Fig. 2. Shows the recognition rate of images at varying FQR levels predicted by the trained MLPs

Once training is completed the MLP can quickly generate FQRs for any input. A system just needs to feed a target image's $64 \times 641 \mathrm{D}$ vector into the trained MLP, and then within $100 \mathrm{~ms}$ an output is generated that is the target image's FQR.

Fig. 2 shows a clear linear relationship between FQRs and recognition success. In order to generate this result, two separate tests were combined and averaged. The first test involved creating 10 separate groups of faces with different recognition rates. Each group was labelled with its real recognition rate in terms of what percentage of the group could be recognized by a recognition method. Then for each group of faces the average FQR was found and recorded against the actual recognition rates. This was our first set of data.

The second test used the opposite approach. 10 separate groups of faces were made using only FQR statistics. Each group was then recognized using a face recognition method and the recognition rate versus the average FQR of that group was recorded. This second set of data was combined with the first set to generate Figure 2. The observed linear relationship implies that the FQR value of an image is a very good approximation of the probability that the tested image will be recognized.

\section{FQR Value Experiments}

In order to rigorously evaluate both FQR and noFQR 11 recognition results in realtime environments with unlimited parameter control, we developed a simulation tool (refer to Fig. 33).

${ }^{1}$ noFQR is the label used for our 2 face recognition methods when implemented without FQR pre-filtering. 


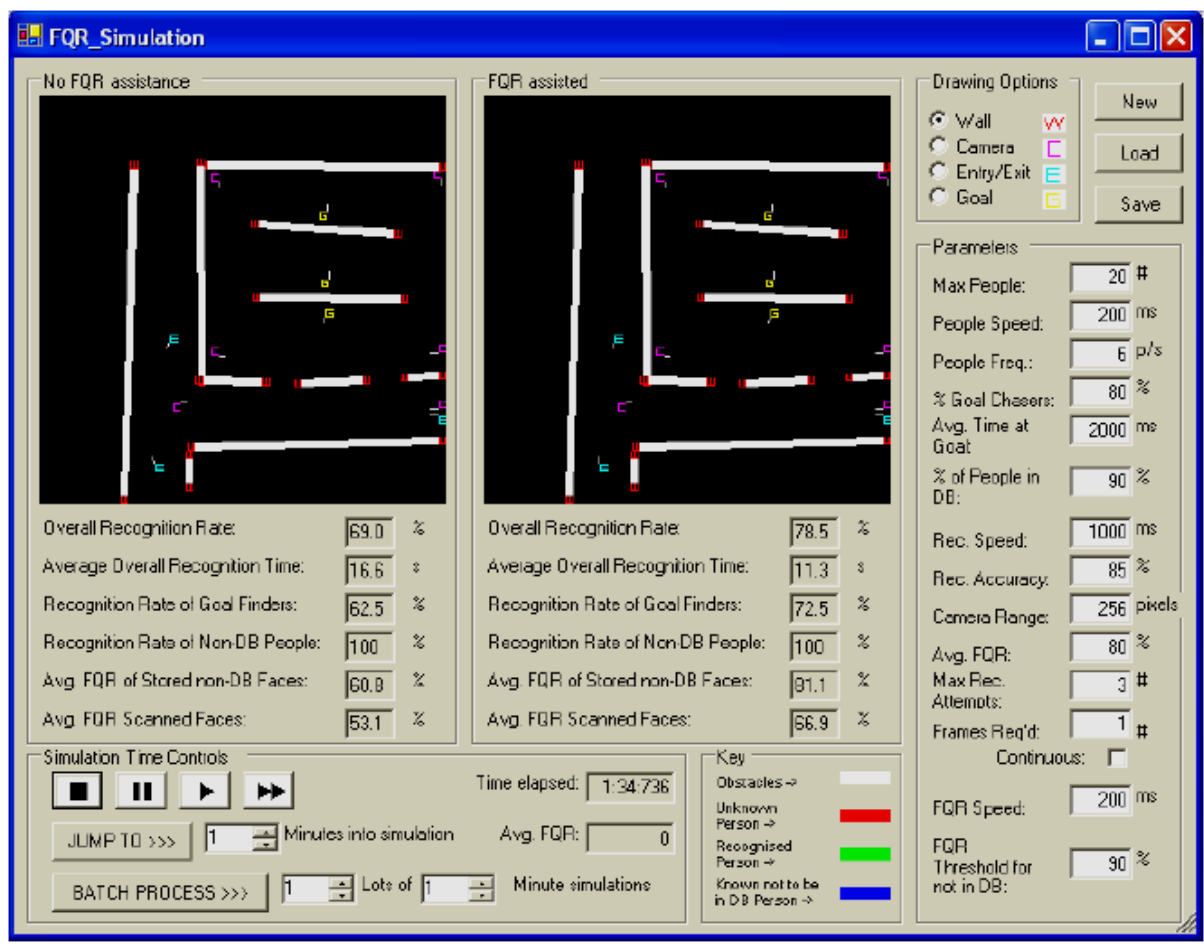

Fig. 3. Simulation Tool: Can simulate any environment that comprises of walls/ tables/chairs (obstacles), entries, exits, sensitive equipment (goals) and cameras. A random crowd is then released into this environment and statistics are recorded for both noFQR (left frame) and FQR face recognition (right frame).

(a)

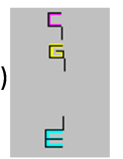

(b)

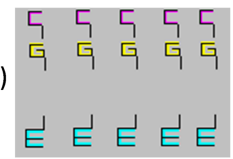

(c)

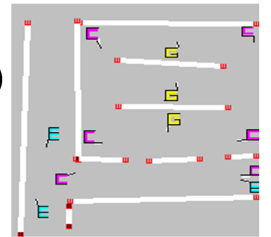

Fig. 4. The 3 standard test environments. (a) A simple access control scenario (b) the same as "a" except this is a public gallery example i.e. train station ticket gates (c) a rough model of one of our postgraduate rooms with a public access hallway and 3 entry/exit points. (Code: $\mathrm{c}=$ camera, $\mathrm{g}=$ goal and $\mathrm{e}=$ entry/exit).

The simulation tool lets us create custom environments (refer to Fig. 4) with many restricted areas, cameras, entry and exit points as well as real-time threaded objects representing people in the scene. Whenever a threaded object moves into the field of view of a camera a real image is generated from our test image set (see below) and placed on the "to do" stack for the environment for every single frame the person stays in the camera's field of view. You can expect 
that with 4 or 5 cameras running at 25 frames per second and hundreds of people objects constantly entering and exiting the scene at random intervals, that the "to do" list can grow extremely rapidly. Only when a person is recognized using our face recognition methods (SFP and PCA), are their image captures removed from the "to do" list. This environment lets us test many hypotheses. Of note however is the effect of changing the maximum population allowed in the scene and the effect of FQR assistance when the recognition methods' speeds were varied. We hypothesize that FQR assisted systems will greatly outperform any noFQR systems.

The images used for these experiments were the same images from the combined database in training except we used 6 images per person and we now also include one of our earlier databases with 120 images of 20 people 14 . This gave us a real-image count of 660 . We also generated 10 virtual images of each real-image giving a total of 7260 images in the complete image set. From this complete image set we extracted three real and two virtual images for 100 people and placed these in our face recognition database. The remaining images (including the images of 10 people not in the face recognition database) were used as test images for the system to find matches of within the face recognition database as the simulation environment presented them. During simulations all of the test images (both real and virtual) were repeatedly used and exposed to random degradations, similar to those used to make the virtual images, in order to generate unlimited test inputs.

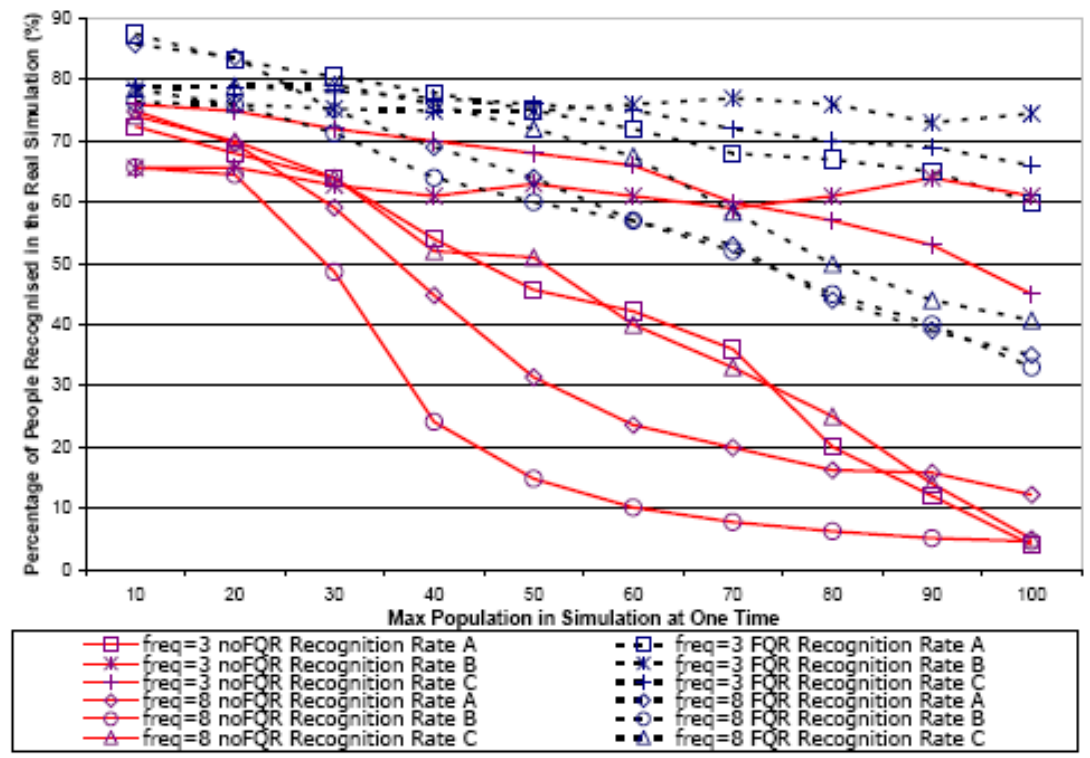

Fig. 5. The effect of max population in the environment and the effect of the frequency with which people arrive in the environment on the Face Recognition Rate 


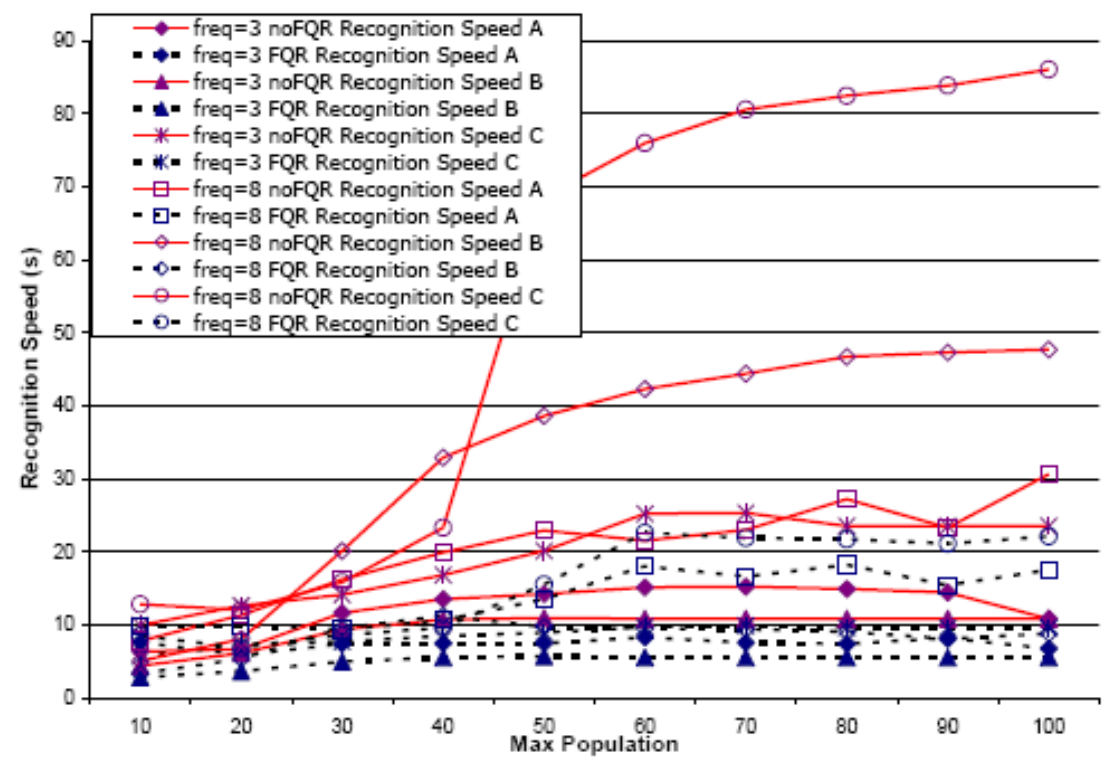

Fig. 6. The effect of max population in the environment and the effect of the frequency with which people arrive in the environment on the Face Recognition Speed

The experiments showed conclusively that using FQR pre-filtering with any face recognition system improved the systems' results dramatically and consistently. The data from Fig. 5 ] and Fig. 6 is the average of both the holistic and the geometric face recognition methods operated in separate tests. The dashed lines representing FQR assisted values show little degradation in performance as the number of people moving through the scene increases, whereas the noFQR methods rapidly degrade. Recognition speed is the average time in seconds it takes to recognize a new arrival in the scene. These graphs show that FQR assistance greatly increases the recognition rate of the face recognition systems. This effect works for both geometric and holistic methods.

It is interesting to note that the noFQR face recognition methods completely fail at relatively small population sizes of 100 people, whereas the FQR methods are almost unaffected. Given that the target applications for FQR assisted methods include airports and train stations where populations sizes will be in the thousands, these initial experiments present a strong case for the FQR tool's inclusion in real-time face recognition approaches.

Fig. 7 and Fig. 8 show that even for very small population sizes of up to 40 people, as soon as a face recognition method's recognition-time approaches a second or more, significant performance degradations are observed. However, when these same systems use FQR pre-filtering the performance is relatively unaffected by recognition-time. This suggests that with the use of FQRs slower but more accurate face recognition methods can be used without effecting overall system speed. This is yet another advantage that FQR pre-filtering can offer. 


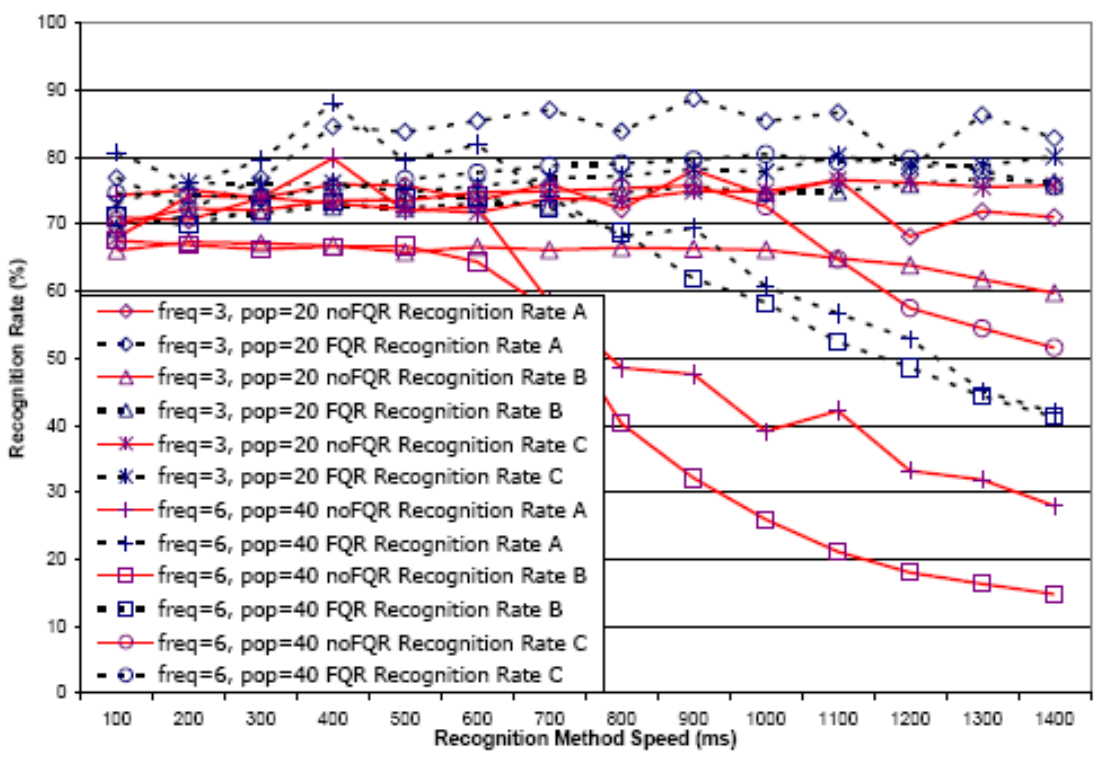

Fig. 7. The effect that the speed of the face recognition component of the system has on the overall system's recognition rate

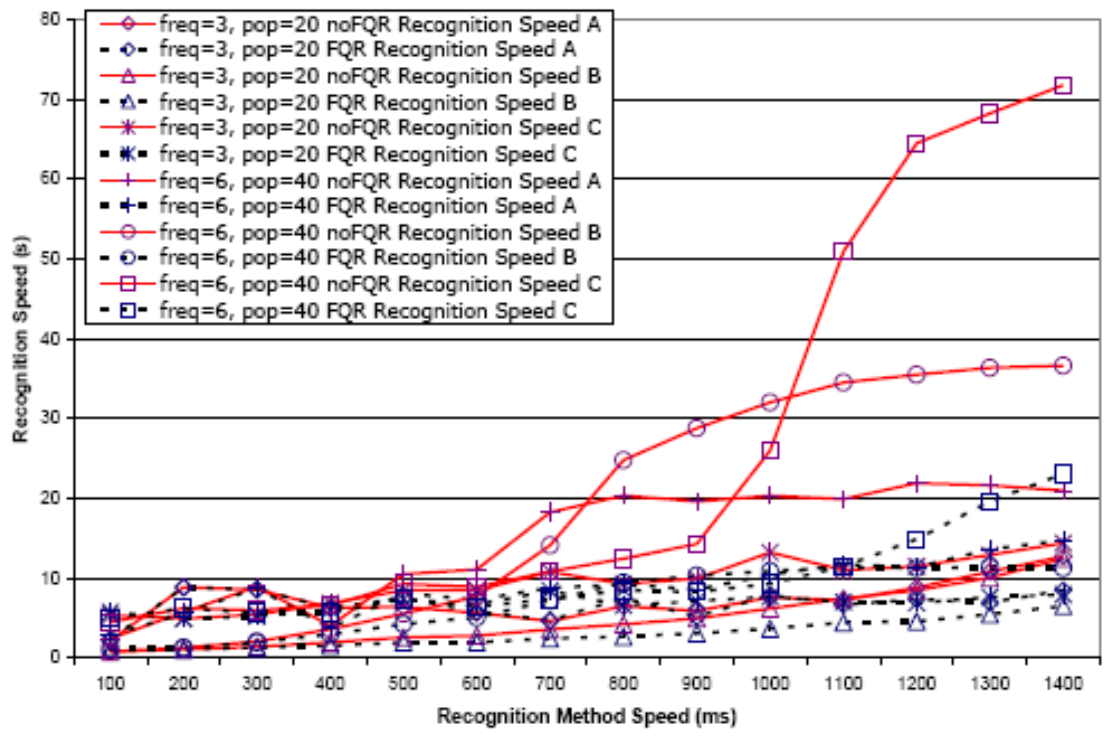

Fig. 8. The effect that the speed of the face recognition component of the system has on the overall system's recognition speed 


\section{Conclusion}

We developed and tested the use of a novel approach for improving face recognition systems. We proved that by intelligently pre-filtering target images, face recognition systems could become much more efficient. A metric was developed called the face quality rating (FQR) that gave an accurate recognition probability value that could be used as a pre-filter in real-time systems. Real-time face recognition experiments demonstrated that on average, an FQR assisted face recognition system will perform better than any other non-FQR assisted face recognition system that uses the same face recognition method and database. By using FQRs any face recognition method can have its accuracy improved by as little as $5 \%$ and as much as $200 \%$, the average results being $20 \%$ to $30 \%$ improvements. The cost of this significant increase in recognition rates was a significant decrease in the time it takes to recognize unknown parties. The recognition time decreases ranged from $5 \%$ to $150 \%$ with $50 \%$ being the average. As such, there are no negative costs involved with implementing our FQR approach. As long as an FQR value can be learned for a face recognition system (i.e. the linear relationship shown in Fig. 2 can be observed with the FQR prediction and recognition results), then improvements in real-time speed and accuracy will occur. Since our FQR training method is generic and can be applied to any face recognition method we are confident that FQRs can be learned for any method.

Future work would involve testing a face recognition method both with and without FQR assistance in a large high traffic public area, such as a university administration building. We would also like to use many different face recognition methods to prove that an FQR value can be learned for any face recognition method.

\section{References}

1. Nastar, C., Mitschke, M.: Real-time face recognition using feature combination. In: Third IEEE International Conference on Automatic Face and Gesture Recognition. Proceedings, 14-16 April 1998, pp. 312-317 (1998)

2. Gorodnichy, D.O.: Video-based framework for face recognition in video. In: The 2nd Canadian Conference on Computer and Robot Vision. Proceedings, 9-11 May 2005, pp. 330-338 (2005)

3. Donald, T.: The Pattern Recognition Basis of Artificial Intelligence, 388 pages. Wiley-IEEE Computer Society Press (1998)

4. Yongsheng, G., Leung, M.K.H.: Face recognition using line edge map. IEEE Transactions on Pattern Analysis and Machine Intelligence 24(6), 764-779 (2002)

5. Ming, Z., Fulcher, J.: Face recognition using artificial neural network group-based adaptive tolerance (GAT) trees. IEEE Transactions on Neural Networks 7(3), 555567 (1996)

6. Quan, Y., Thangali, A., Sclaroff, S.: Face Identification by a Cascade of Rejection Classifiers. In: IEEE Computer Society Conference on Computer Vision and Pattern Recognition, 20-26 June, vol. 3, p. 152 (2005)

7. Shan, D., Ward, R.: Wavelet-based illumination normalization for face recognition. In: IEEE International Conference on Image Processing, ICIP 2005, 11-14 September, vol. 2, pp. II 954-II 957 (2005) 
8. Quanren, X., Jaynes, C.: Mugshot database acquisition in video surveillance networks using incremental auto-clustering quality measures. In: IEEE Conference on Advanced Video and Signal Based Surveillance. Proceedings, 21-22 July 2003, pp. 191-198 (2003)

9. Subasic, M., Loncaric, S., Petkovic, T., Bogunovic, H., Krivec, V.: Face image validation system: Image and Signal Processing and Analysis. In: Proceedings of the 4th International Symposium on Image and Signal Processing and Analysis, ISPA 2005, 15-17 September, pp. 30-33 (2005)

10. Bennett Jr., H.H., Campbell Jr., R.L., Younan, N.H.: Use of rejection class to enhance airborne imagery classification. In: Conference Record of the Thirty-Third Asilomar Conference on Signals, Systems, and Computers, 1999, 24-27 October 1999, vol. 2, pp. 845-848 (1999)

11. Ming-Hsuan, Y., Kriegman, D.J., Ahuja, N.: Detecting faces in images: a survey. IEEE Transactions on Pattern Analysis and Machine Intelligence 24(1), 34-58 (2002)

12. Lech, M., Hua, Y.: Vector quantization of images using neural networks and simulated annealing. In: Proceedings of the 1991 IEEE Workshop on Neural Networks for Signal Processing [1991], 30 September-1 October 1991, pp. 552-561 (1991)

13. Sim, T., Baker, S., Bsat, M.: The CMU Pose, Illumination, and Expression Database. IEEE Transactions on Pattern Analysis and Machine Intelligence 25(12), 1615-1618 (2003)

14. Axnick, K., Jarvis, R.: Face and Pose Recognition for Robotic Surveillance. In: Proceedings of the 2005 Australasian Conference on Robotics and Automation (2005)

15. Axnick, K., Ng, K.: Fast Face Recognition: Image and Vision Computing Conference On, New Zealand University of Otago, Dunedin, 28-29 November (2005)

16. Anbang, X., Xin, J., Yugang, J., Ping, G.: Complete Two-Dimensional PCA for Face Recognition. In: 18th International Conference on Pattern Recognition, ICPR 2006, vol. 3, pp. 481-484 (2006) 